TAO, Vol. 16, No. 1, 251-271, March 2005

\title{
A Study of Recurrence Models of Earthquakes in Taiwan
}

\author{
Chien-Ping Lee ${ }^{1, *}$ and Yi-Ben Tsai ${ }^{1}$ \\ (Manuscript received 23 April 2004, in final form 13 January 2005)
}

\begin{abstract}
Taiwan has one of the most complete instrumental catalogs of earthquakes in the world. Data from the Taiwan Central Weather Bureau and the Institute of Earth Sciences, Academia Sinica provide a fairly complete catalog of earthquakes with magnitude greater than $\mathbf{5 . 0}$ since $\mathbf{1 9 0 0}$, and with magnitude greater than 3.0 since 1973 . We have made a study of recurrence models of earthquakes in Taiwan using this catalog with magnitude $\geq 4.0$ from 1973 to 2001 and magnitude $\geq 5.0$ from 1900 to 2001. We first compared four recurrence models (i.e., exponential, Weibull, Gamma, and lognormal models) utilizing the data in fifteen circular areas of Taiwan. The model parameters were determined through data fitting by the method of moments and the method of maximum likelihood. It was found that the method of moments tends to give better fitting. Results further show that the Gamma model using the method of moments usually gives better fitting to the data than other recurrence models for all magnitudes. The two parameters ( $c$ and $r$ ) of the Gamma model are subsequently used to show the characteristics of seismicity patterns. For example, large $c$ values appear in eastern Taiwan because of more frequent earthquakes there. Small $r$ values are found in central Taiwan because of clustering of numerous aftershocks following the Chi-Chi earthquake. These parameters of the Gamma recurrence model are useful for estimating probabilistic seismic hazards and for forecasting the probability of future earthquakes in different areas of Taiwan. For example, the probability for a magnitude $\geq 6.0$ in the whole of Taiwan reaches 70 percent at a recurrent time of about 200 days.
\end{abstract}

(Key words: Seismicity, Earthquake catalog, Recurrence model, Gamma model, Weibull model)

\footnotetext{
${ }^{1}$ Institute of Geophysics, National Central University, Chung-Li, Taiwan, ROC

* Corresponding author address: Mr. Chien-Ping Lee, Institute of Geophysics, National Central University, Chung-Li, Taiwan, ROC;E-mail: cplee@geps.gep.ncu.edu.tw
} 


\section{INTRODUCTION}

Statistical studies of earthquake occurrences have frequently been carried out since the early years of seismology. In order to obtain reliable results from a statistical analysis, sufficient amount of high-quality data is necessary. The data are usually taken from earthquake catalogs, but many of the existing catalogs are inhomogeneous and incomplete. Instrumental seismic observations in Taiwan started at 1898. An earthquake catalog with uniform $\mathrm{M}_{\mathrm{L}}$ magnitude for one hundred years in Taiwan was developed by Shin (1993) and Yeh et al. (1995) by combining the catalogs of the Taiwan Central Weather Bureau (CWB) and of the Institute of Earth Sciences (IES), Academia Sinica. The composite catalog has become one of the most complete instrumental catalogs of earthquakes in the world. For this reason, we have utilized these excellent catalog data to find suitable recurrence models of earthquakes in Taiwan.

There are many studies about recurrence models of earthquakes in the literature. The Weibull distribution (Hagiwara 1974; Sykes and Nishenko 1984; Utsu 1984; Rikitake 1991; Parvez and Ram 1997), Gamma distribution (Udías and Rice 1975; Utsu 1984), and lognormal distribution (Nishenko and Bulland 1987; Goes 1996; Shimazaki 2002) are typical distributions that researchers used previously. The exponential distribution (Poisson process) is a special case of the Weibull distribution or Gamma distribution. Five recurrence models were reviewed by Utsu (2002), including the exponential, Weibull, lognormal, Gamma, and doubly exponential distributions. Besides these pure mathematical models, other novel models for recurrence of large earthquakes have been developed recently (Matthews et al. 2002; Gómez and Pacheco 2004).

Since heavy damage and loss of life are often caused by large earthquakes, we will focus on moderate to large earthquakes in this study. We seek to determine parameters for recurrence models using the Taiwan earthquake catalog and to find a best-fit recurrence model for the data in Taiwan. The results clearly show distinct characteristics of earthquake occurrence in different regions of Taiwan.

\section{DATA}

There have been many disastrous earthquakes in Taiwan. The instrumental period for earthquake observation in Taiwan began in 1898. The Central Meteorological Observatory (CMO, the predecessor of Taiwan Central Weather Bureau) deployed several seismic stations to observe earthquakes. The Taiwan Telemetered Seismograph Network (TTSN) was deployed by the IES, Academia Sinica in 1973. Since 1991, the TTSN has merged with the original CWB network then been upgraded and densified to become the advanced Central Weather Bureau Seismic Network (CWBSN). There were several earthquake catalogs in Taiwan in the early periods (Cheng and Yeh 1989; Wang 1992); and the magnitude scales of such catalogs were not uniform. Shin (1993) developed a method to determine local magnitude from simulated Wood-Anderson seismograms of short-period seismograms, called $\mathrm{M}_{\mathrm{L}(\mathrm{Shin})}$. Since then the $\mathrm{M}_{\mathrm{L}(\mathrm{Shin})}$ has become the standard local magnitude for the CWB earthquake catalog. In order to unify previous Taiwan earthquake catalogs, Yeh et al. (1995) converted various mag- 
nitude scales used in different periods into $\mathrm{M}_{\mathrm{L}(\mathrm{Shin})}$. We shall use this unified earthquake cata$\log$ that dates back over the past one hundred years for our present study. Hereafter we use $\mathrm{M}_{\mathrm{L}}$ to denote $\mathrm{M}_{\mathrm{L}(\mathrm{Shin})}$.

In order to obtain reliable results from any statistical analysis, a sufficient amount of highquality data is required. The data are usually taken from earthquake catalogs. Unfortunately, many of the existing catalogs are inhomogeneous and incomplete. Like most researchers, we use the Gutenberg-Richter relationship (Gutenberg and Richter 1944, 1949) to check the completeness of the unified CWB earthquake catalog. Figure 1 shows such plots for three periods based on the CWB earthquake catalog in Taiwan. The circle denotes the cumulative logarith-

Taiwan

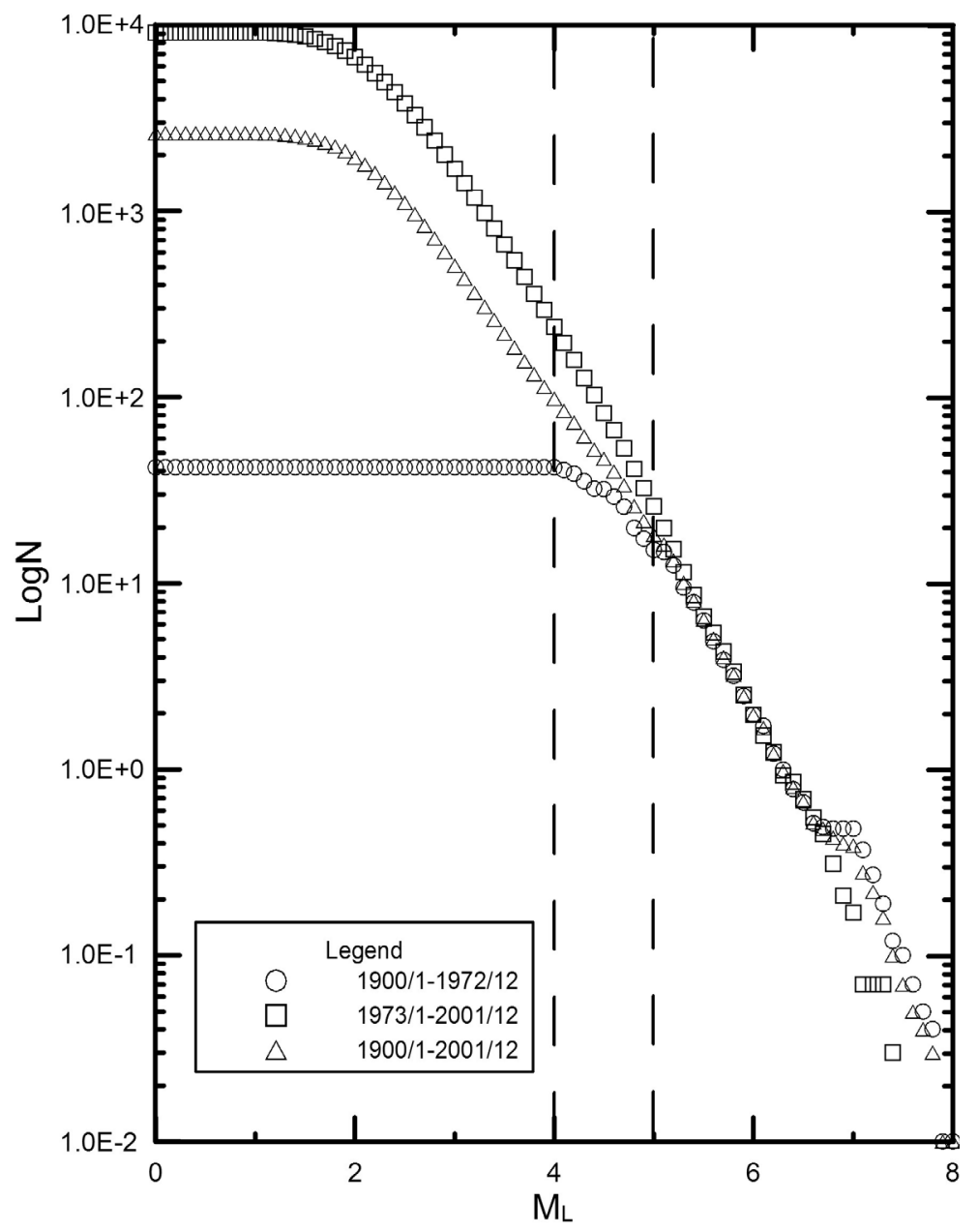

Fig. 1. The relationship between earthquake magnitude and numbers per year in Taiwan. 
mic number of earthquakes versus $M_{L}$ from 1900 to 1972 . The square denotes the cumulative logarithmic numbers of earthquakes versus $\mathrm{M}_{\mathrm{L}}$ from 1973 to 2001. The triangle denotes the cumulative logarithmic number of earthquakes versus $M_{L}$ from 1900 to 2001. From the figure, we can see the unified catalog, based on the Seismological Bulletins of the CWB and the Catalogs of Earthquakes in Taiwan by the IES of Academia Sinica, can provide a rather complete catalog of earthquakes with $\mathrm{M}_{\mathrm{L}}$ magnitude greater than 5.0 since 1900 and with $\mathrm{M}_{\mathrm{L}}$ magnitude greater than 3.0 since 1973. In this study we shall use this earthquake catalog to find suitable recurrence models. Considering the completeness of earthquake catalog (Fig. 1), we decide to select the earthquakes with $M_{L}$ magnitude $\geq 4.0$ for the period from 1973 to 2001 and $\mathrm{M}_{\mathrm{L}}$ magnitude $\geq 5.0$ for the period from 1900 to 2001 .

\section{EARTHQUAKE RECURRENCE MODELS}

There are several general statistical models frequently used for earthquake recurrence (Utsu 1984, 2002). In this study we start with four recurrence models (Utsu 2002) to analyze the CWB earthquake catalog. Given $w(t)$ as the probability density function, $\phi(t)$ as the cumulative probability function, and $t$ as the interevent time, these models can be expressed as follows:

Exponential (Poisson) model:

$$
\begin{aligned}
& w(t)=\lambda \exp (-\lambda t) \quad \lambda>0, \\
& \phi(t)=1-\exp (-\lambda t) .
\end{aligned}
$$

Weibull model:

$$
\begin{aligned}
& w(t)=\alpha \beta t^{\beta-1} \exp \left(-\alpha t^{\beta}\right) \quad \alpha>0, \beta>0, \\
& \phi(t)=1-\exp \left(-\alpha t^{\beta}\right) .
\end{aligned}
$$

Gamma model:

$$
\begin{aligned}
& w(t)=c(c t)^{r-1} \exp (-c t) / \Gamma(r) c>0, r>0, \\
& \phi(t)=\Gamma(r, c t) / \Gamma(r),
\end{aligned}
$$

where $\Gamma(k, x)$ represents the incomplete gamma function of the second kind, i.e., $\Gamma(k, x)=\int_{x}^{\infty} e^{-u} u^{k-1} d u$.

Lognormal model: 


$$
\begin{aligned}
& w(t)=1 /(\sqrt{2 \pi} \sigma) \exp \left\{-(\ln t-m)^{2} / 2 \sigma^{2}\right\} \mathrm{m}>0, \sigma>0, \\
& \phi(t)=\Phi\{(\ln t-m) / \sigma\},
\end{aligned}
$$

where $\Phi(x)$ represents the error integral defined by $\Phi=(1 / \sqrt{2 \pi}) \int_{-\infty}^{x} e^{-u^{2} / 2} d u$.

Note that the exponential distribution (Poisson process) is a special case of the Weibull distribution ( $\beta=1$ ), or the Gamma distribution $(r=1)$. If $\beta<1$ or $r<1$, the earthquakes have a tendency to cluster in time. If $\beta>1$ or $r>1$, the earthquakes have a tendency of intermittent occurrence.

\section{ANALYSIS}

We divide Taiwan into 15 circular areas by using CWB short-period seismic stations as centers with $50 \mathrm{~km}$ radii, except PUL $\left(24.0^{\circ} \mathrm{N}, 121.0^{\circ} \mathrm{E}\right)$, which is not a seismic station. Figure 2 shows earthquakes with $M_{L}$ magnitude $\geq 4.0$ in our study area from 1900 to 2001 . The completeness of earthquake data for these areas is checked in Fig. 3. As expected, the GutenbergRichter relationship is not as robust in some circular areas as it is for the whole Taiwan because of fewer earthquakes. In eastern Taiwan, the Gutenberg-Richter relationships are good, such as in the ILA, ENA, HWA, TWF1, and TTN areas. Because many large aftershocks occurred after the Chi-Chi earthquake ( $\mathrm{M}_{\mathrm{L}}$ 7.3, 17:47, 20 September 1999, Universal Time), the Gutenberg-Richter relationships jump up for $\mathrm{M}_{\mathrm{L}}$ magnitude greater than 5.0 in the TCU, PUL, and YUS areas. The relationship is poor in the HSN and KAU areas. Even though the Gutenberg-Richter relationship is not good in some areas, the earthquakes with $\mathrm{M}_{\mathrm{L}}$ magnitude $\geq 5.0$ are basically complete for 1900 to 2001, and the earthquakes with $\mathrm{M}_{\mathrm{L}}$ magnitude $\geq 4.0$ are complete for 1973 to 2001 . Therefore, we divide the earthquake catalog into subsets with $\mathrm{M}_{\mathrm{L}}$ magnitudes greater than 4.0, 4.5, 5.0, 5.5, and 6.0 subsequently. Considering the completeness of earthquake catalog, we decide to select the earthquakes with magnitude $\geq 4.0$ in each area from 1973 to 2001 and magnitude $\geq 5.0$ from 1900 to 2001 . We calculated the interval time and counted the number of earthquakes. Then we normalized the number of earthquakes to get the probability of an earthquake occurrence. These observation results are used to construct the recurrence models. In the end, we compared the observed results among the four recurrence models.

We used both the method of maximum likelihood and the method of moments to estimate the model parameters $(\lambda, \alpha, \beta, c, r, m, \sigma)$ of the four recurrence models, e.g., exponential (Poisson), Weibull, Gamma and lognormal model (Utsu 1984). In order to choose the best model, we calculated the root-mean-squares (RMS) of the residuals between data value and the recurrence model estimate. This formed the selection criteria. Then we used the parameters of the best fitting model to study the seismicity characteristics of each area. 


\section{Taiwan}

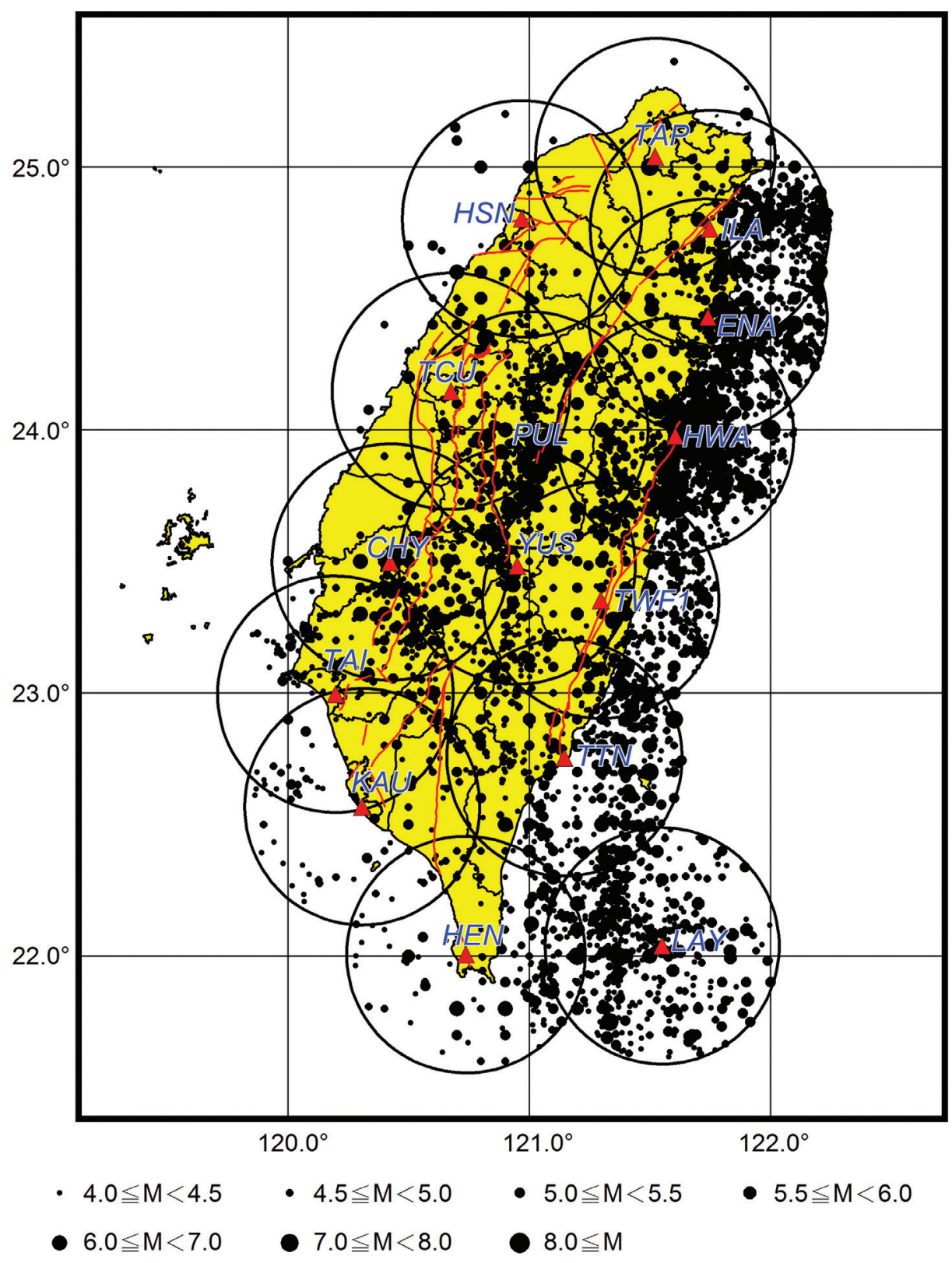

Fig. 2. Seismicity of Taiwan with magnitude $\geq 4.0$ in fifteen circular areas from 1900 to 2001 . The triangles denote the short-period seismic stations. The red lines distributed in Taiwan show active faults. 

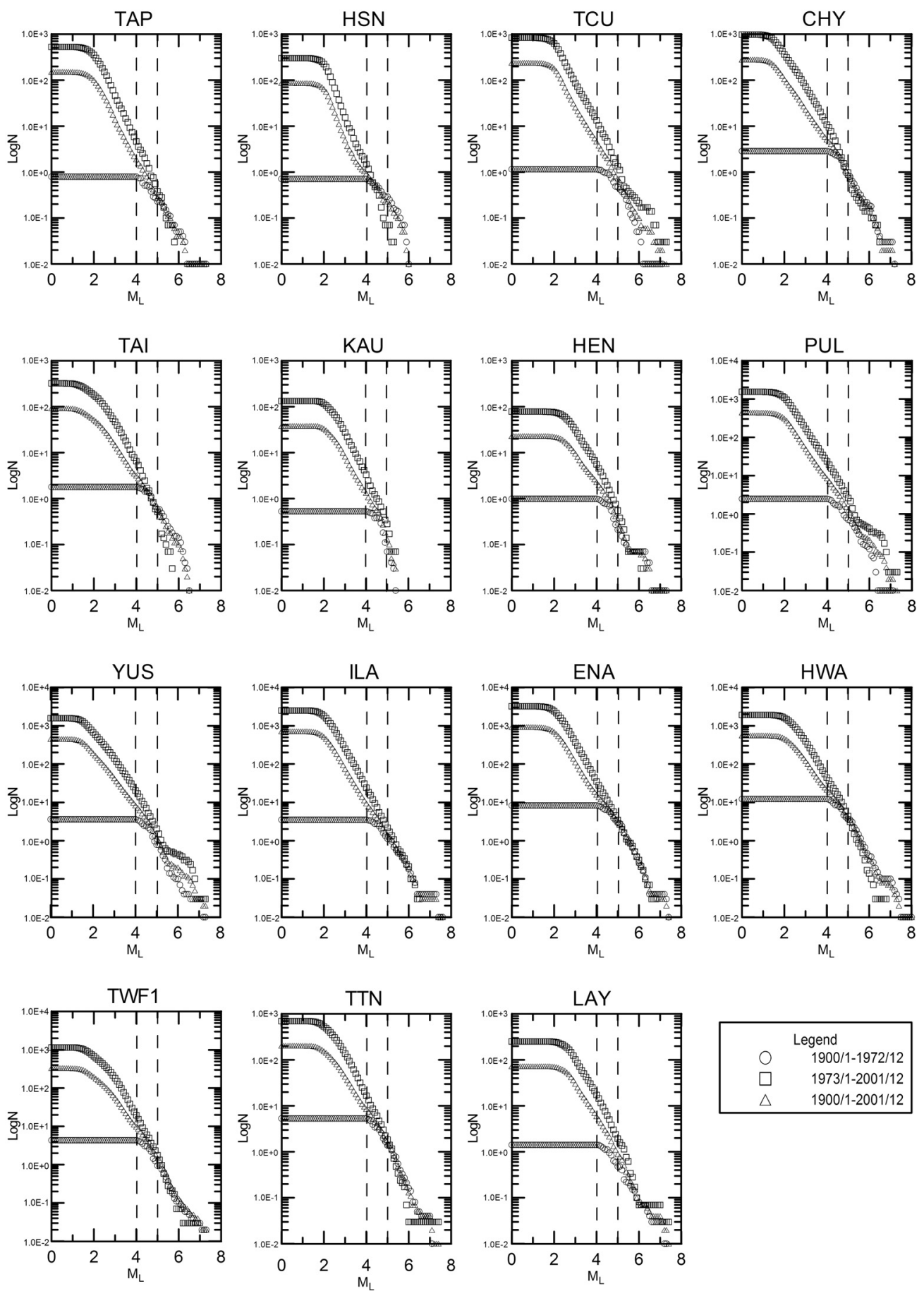

Fig. 3. The relationship between earthquake magnitude and number per year in fifteen circular areas. 


\section{RESULTS}

As mentioned previously, we have divided Taiwan into fifteen areas in this study. Here we just use the case for the Nanao (ENA) area as an example to demonstrate our results. The events in this area are more numerous than in other areas. Figure 4 shows the seismicity of the Nanao area with $M_{L}$ magnitude $\geq 4.0$ from 1900 to 2001. The triangle denotes each short period seismic station. The earthquakes inside the circular boundary are used here to calculate

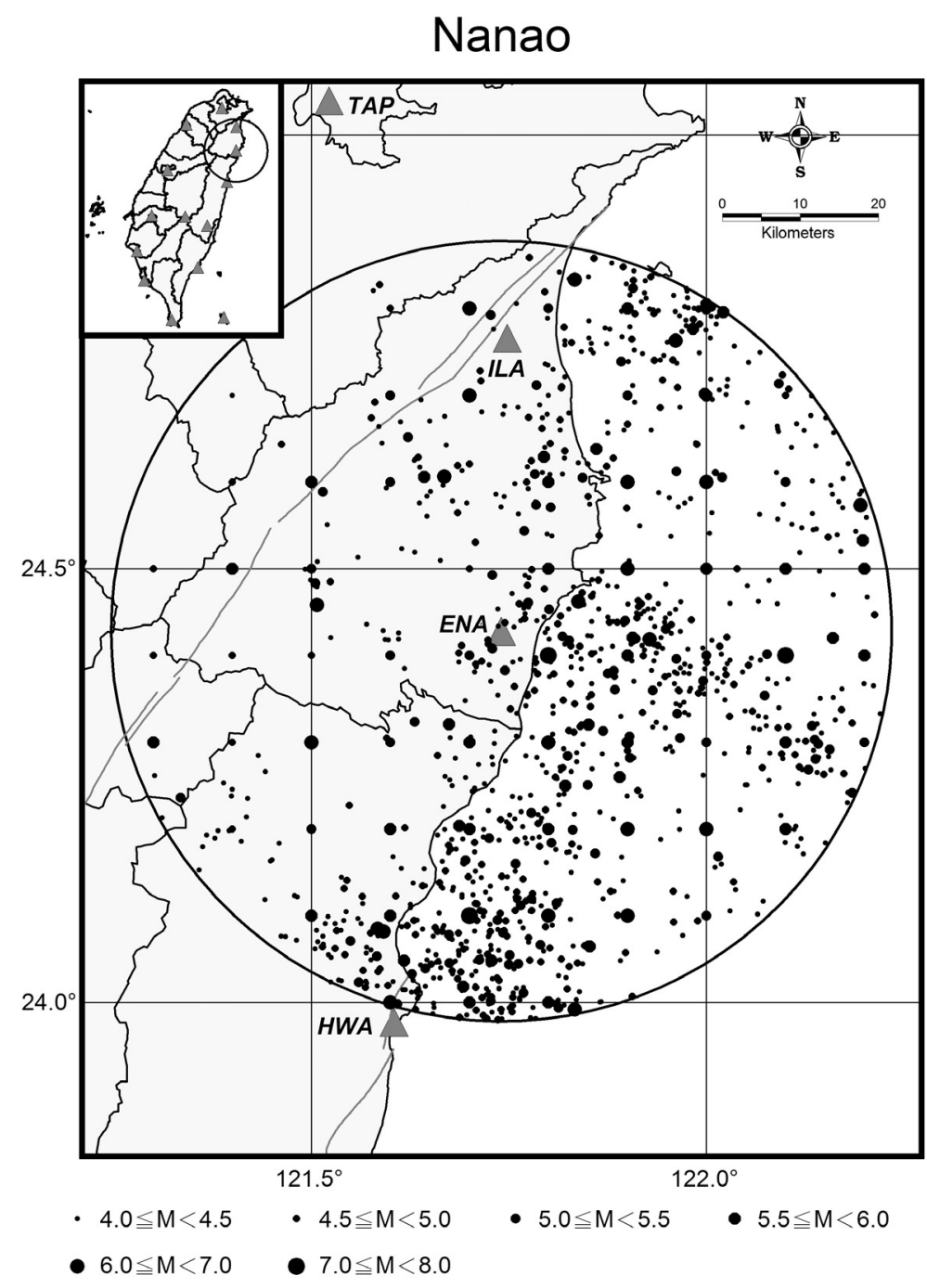

Fig. 4. Seismicity of the Nanao (ENA) area with magnitude $\geq 4.0$ from 1900 to 2001. The triangle denotes the short-period seismic station. The lines distributed in Taiwan show active faults. 
interval time and probability. Figure 5 shows the probability versus interval time for different magnitude ranges. The curves from left to right are for $\mathrm{M}_{\mathrm{L}}$ magnitudes greater than $4.0,4.5,5.0,5.5$, and 6.0, respectively. The probability of occurrence of small earthquakes is higher than that of large earthquakes for the same interval time. The data plots become smooth when the data set is large enough.

Figure 6 shows a comparison of the observed data for $M_{L}$ magnitude $\geq 5.0$ with the four recurrence models, including Poisson, Weibull, Gamma, and lognormal models, using the method of maximum likelihood. The Gamma model and Weibull model apparently fit the data better than the other two models. All recurrence models fit poorly when the time interval is

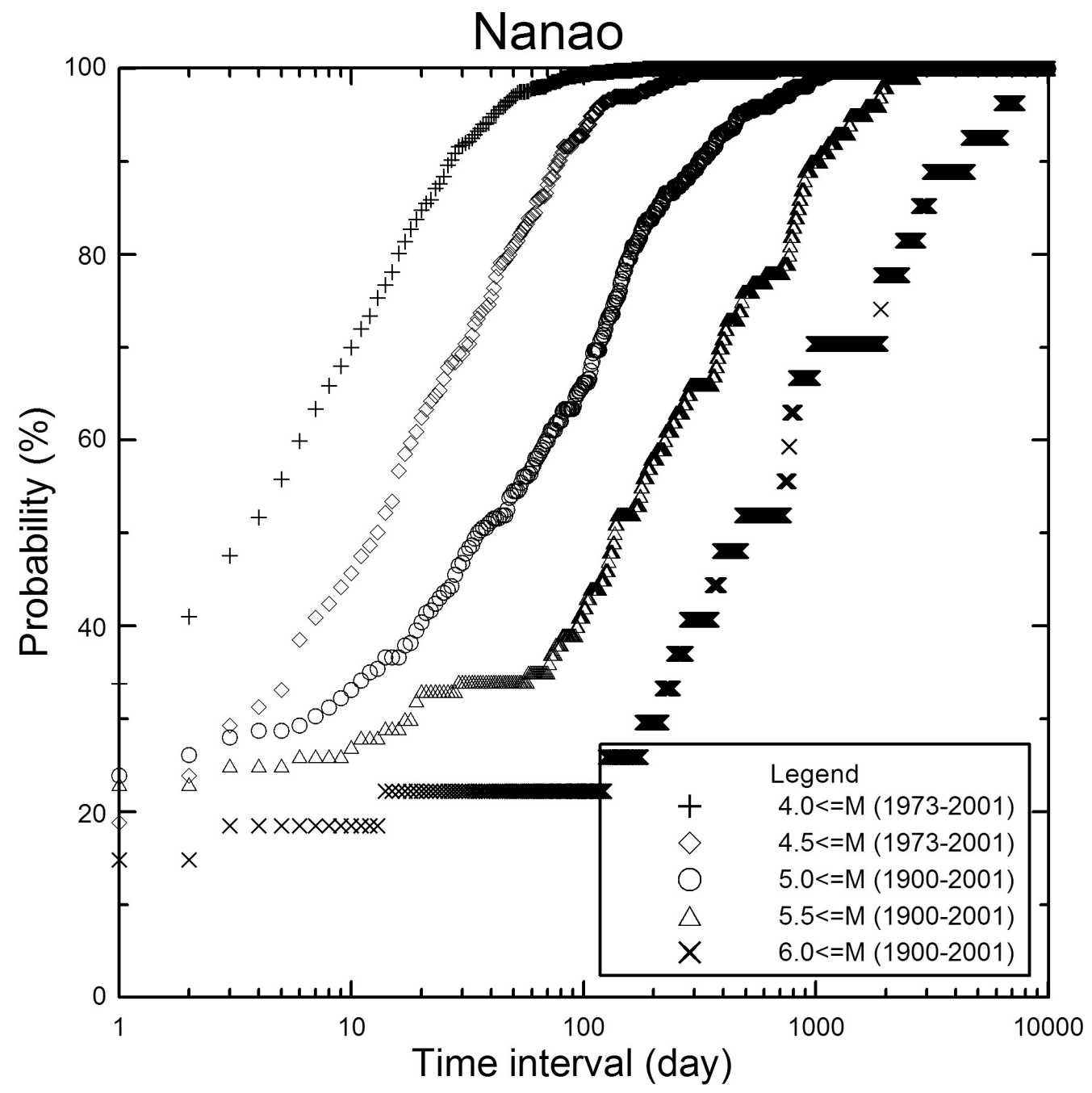

Fig. 5. The probability and the interval time relationship for different magnitude ranges in the Nanao area. 
less than 100 days. It is contaminated by aftershocks because the interval is short and interevent distance is less than 100 kilometers (The diameter of each circular area is $100 \mathrm{~km}$ ). Similar results using the method of moments are shown in Fig. 7, where the Gamma model and Weibull model again fit the observed data better than the other two models. By comparing the Gamma model using both the methods of moments and of maximum likelihood, we conclude that the Gamma model derived from fitting by the method of moments is better than the Gamma model derived from fitting by the method of maximum likelihood. Therefore, we decided to deter-

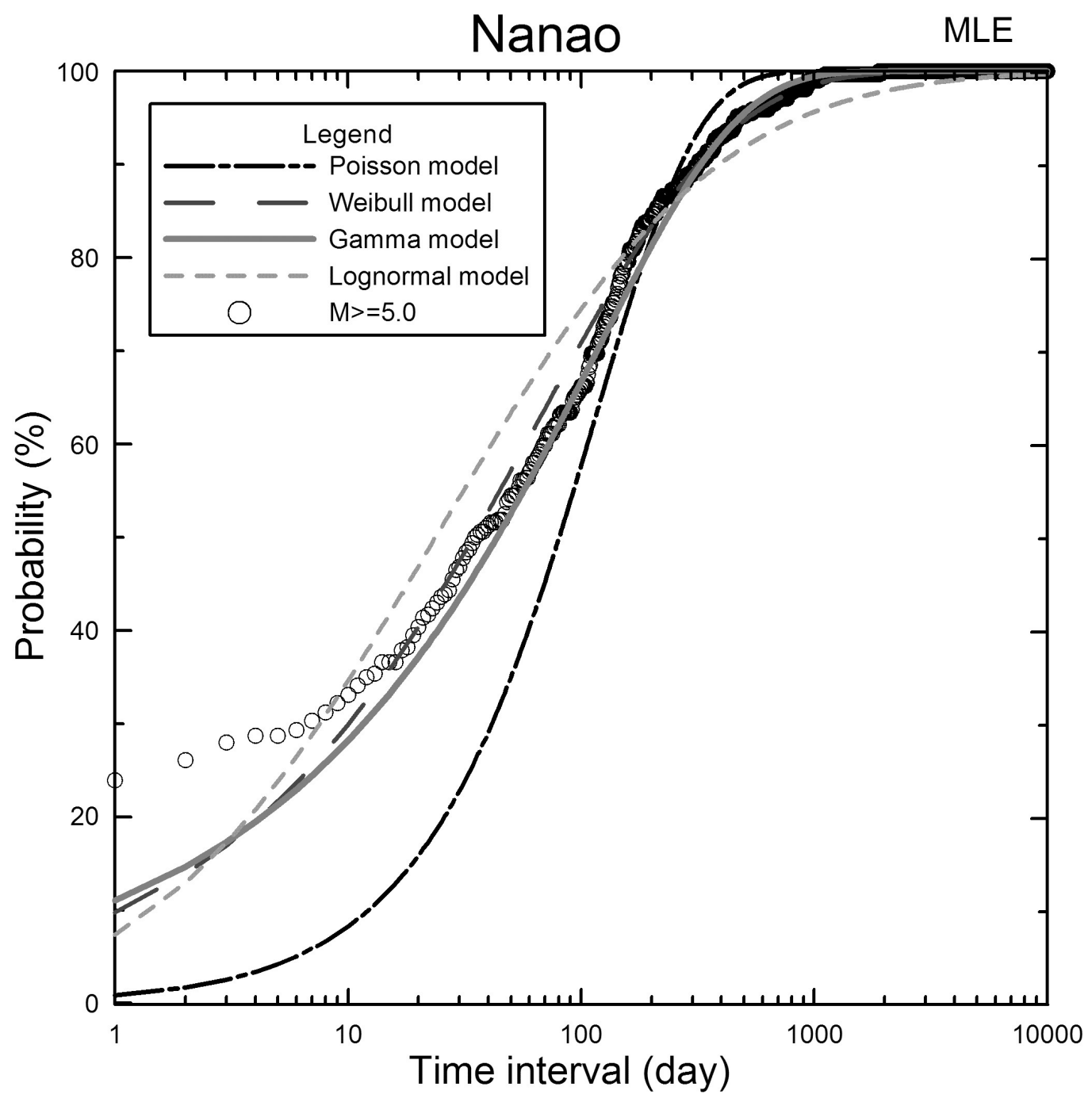

Fig. 6. The probability and the interval time relationship for magnitude $\geq 5.0$ in the Nanao area. Four recurrence models are compared using the method of maximum likelihood. 


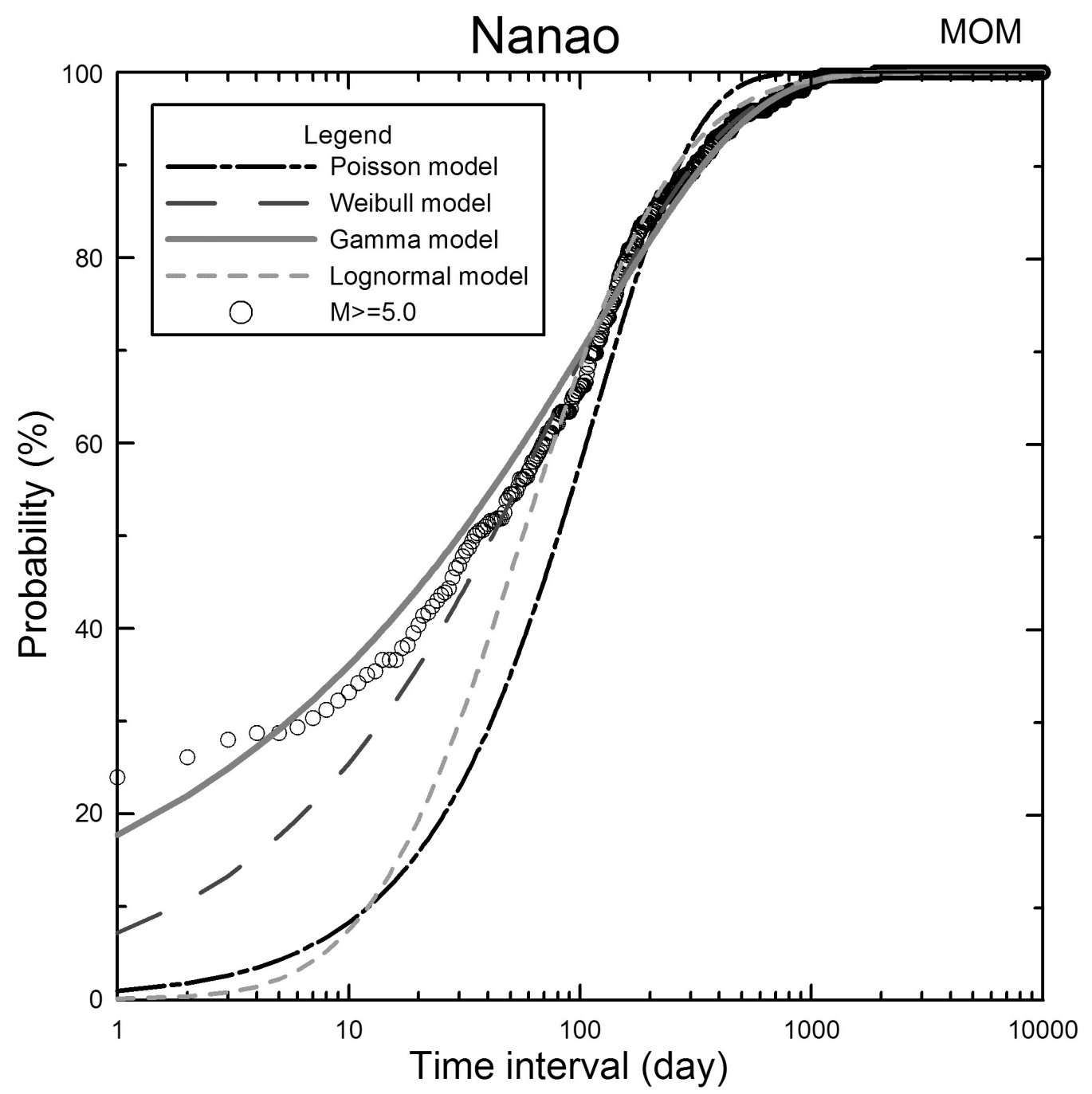

Fig. 7. The probability and the interval time relationship for magnitude $\geq 5.0$ in the Nanao area. Four recurrence models are compared using the method of moments.

mine the Gamma model parameters using the method of moments for data fitting.

Table 1 lists the root-mean-squares of residuals for the four models using the method of moments for data fitting in the fifteen areas. The $\mathrm{M}_{\mathrm{L}}$ magnitudes are greater than 5.0 in this case. Results show that the Gamma model and Weibull model fit significantly better with the data than the other two models in all fifteen areas. Furthermore, the Gamma model is slightly better than the Weibull model. As a result, we calculated the parameters of the Gamma model by the method of moments for data fitting for $\mathrm{M}_{\mathrm{L}}$ magnitudes $\geq 4.0,4.5,5.0,5.5$, and 6.0, 
Table 1. The root-mean-squares of residuals for the four recurrence models using the method of moments for data fitting for magnitude $\geq 5.0$ in the fifteen areas.

\begin{tabular}{|c|c|c|c|c|}
\hline Station & Gamma & Weibull & Lognormal & Poisson \\
\hline TAP & 0.3029 & 0.3024 & 0.2976 & 0.3037 \\
\hline HSN & 0.2389 & 0.2489 & 0.2842 & 0.294 \\
\hline TCU & 0.0913 & 0.1142 & 0.1504 & 0.1889 \\
\hline CHY & 0.0912 & 0.1028 & 0.123 & 0.1411 \\
\hline TAI & 0.1217 & 0.1278 & 0.1463 & 0.1435 \\
\hline KAU & 0.258 & 0.2608 & 0.2823 & 0.2745 \\
\hline HEN & 0.1556 & 0.1458 & 0.1466 & 0.1577 \\
\hline ILA & 0.1207 & 0.1203 & 0.12 & 0.1247 \\
\hline ENA & 0.0352 & 0.032 & 0.0505 & 0.0694 \\
\hline HWA & 0.026 & 0.0343 & 0.0532 & 0.0682 \\
\hline TWF1 & 0.056 & 0.0546 & 0.0733 & 0.0973 \\
\hline TTN & 0.0539 & 0.0533 & 0.0756 & 0.0908 \\
\hline PUL & 0.071 & 0.0892 & 0.1164 & 0.1581 \\
\hline YUS & 0.0557 & 0.0744 & 0.1046 & 0.1312 \\
\hline LAY & 0.0923 & 0.0821 & 0.1003 & 0.1398 \\
\hline
\end{tabular}

respectively. Figure 8 shows the fitting of observed data with the Gamma model using the method of moments for different magnitude ranges. The fit is good when the time interval is greater than 10 days for $\mathrm{M}_{\mathrm{L}}$ magnitude $\geq 4$.0. As the magnitude increases, the fitting error also increases with interval time. This is due to aftershock contamination, especially for $\mathrm{M}_{\mathrm{L}}$ magnitude $\geq 6.0$. The model curve underestimates the data for $M_{L}$ magnitude $\geq 6.0$ when the interval time is less than 100 days. The other reason is that the data set for magnitude $\geq 4.0$ is larger than that for magnitude $\geq 6.0$. The fitting curves in Fig. 8 show the recurrence of earthquakes with different magnitudes in the Nanao area. We can see that at a probability of 70 percent the recurrence interval times are $10,30,100,400$, and 1500 days corresponding to magnitudes greater than 4.0, 4.5, 5.0, 5.5, and 6.0, respectively. This not only shows earthquakes occur more frequently in smaller magnitudes, but also quantifies the probability of the occurrence of an earthquake as a percentage for different magnitudes.

There are two parameters ( $c$ and $r$ ) in the Gamma model. These two parameters affect the curve shape of the Gamma model. As the value of $c$ increases, the curve moves toward the left indicating more earthquakes occurring at short interval times. That is earthquakes will occur 


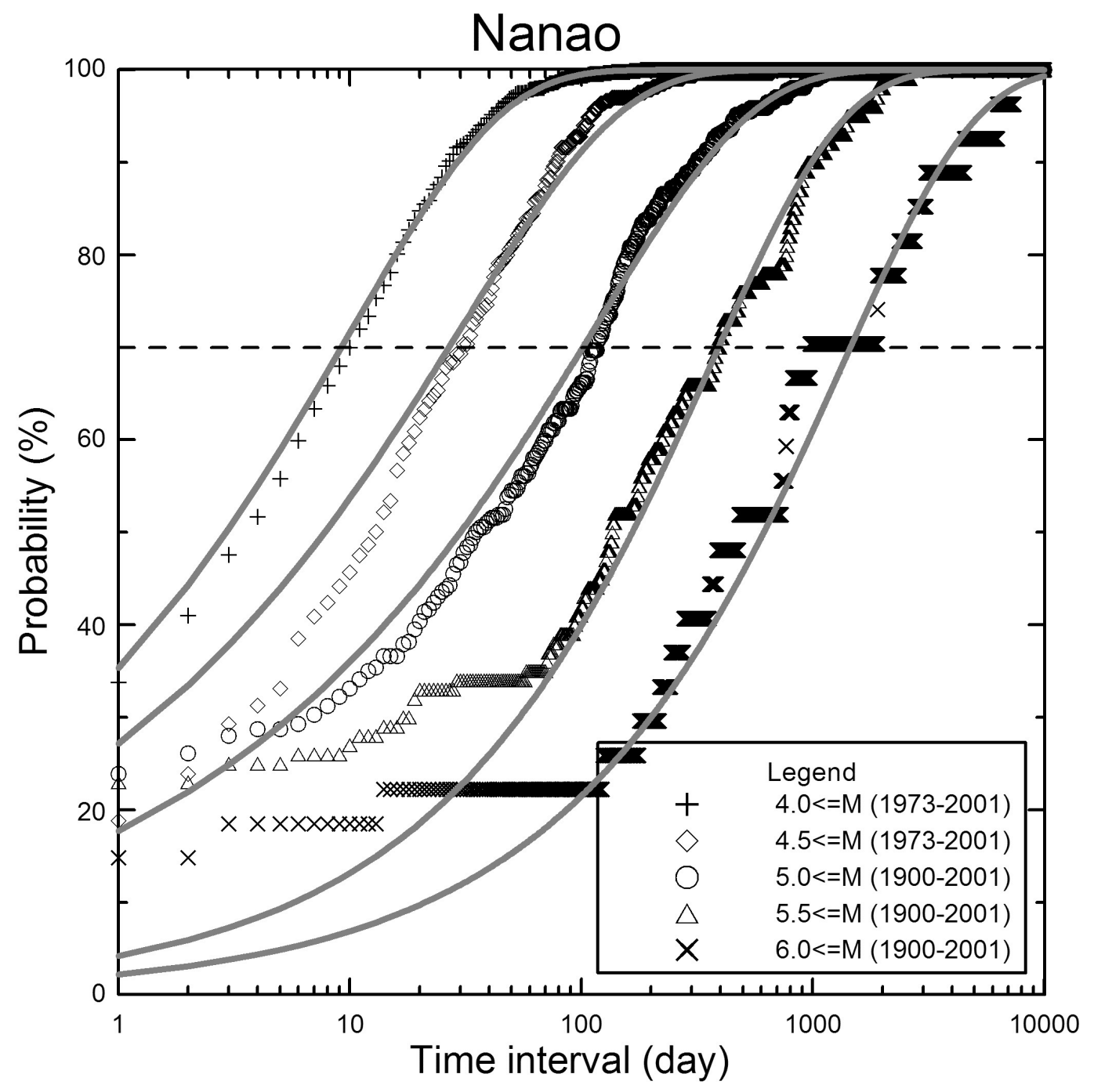

Fig. 8. The relationship of the probability and interval time with different magnitude ranges in the Nanao area. The corresponding fittings are from the Gamma model using the method of moments.

more frequently. Conversely, a small value of $c$ means earthquakes will occur less frequently. The other parameter $r$ affects the slope of the curve. As the value of $r$ decreases, the slope of the curve becomes gentler. This implies that there are many aftershocks occurring at short interval times. Conversely, should the slope of the curve increase sharply then there are no aftershocks or earthquake swarms.

We calculated the two parameters of the Gamma model for earthquakes with magnitudes 


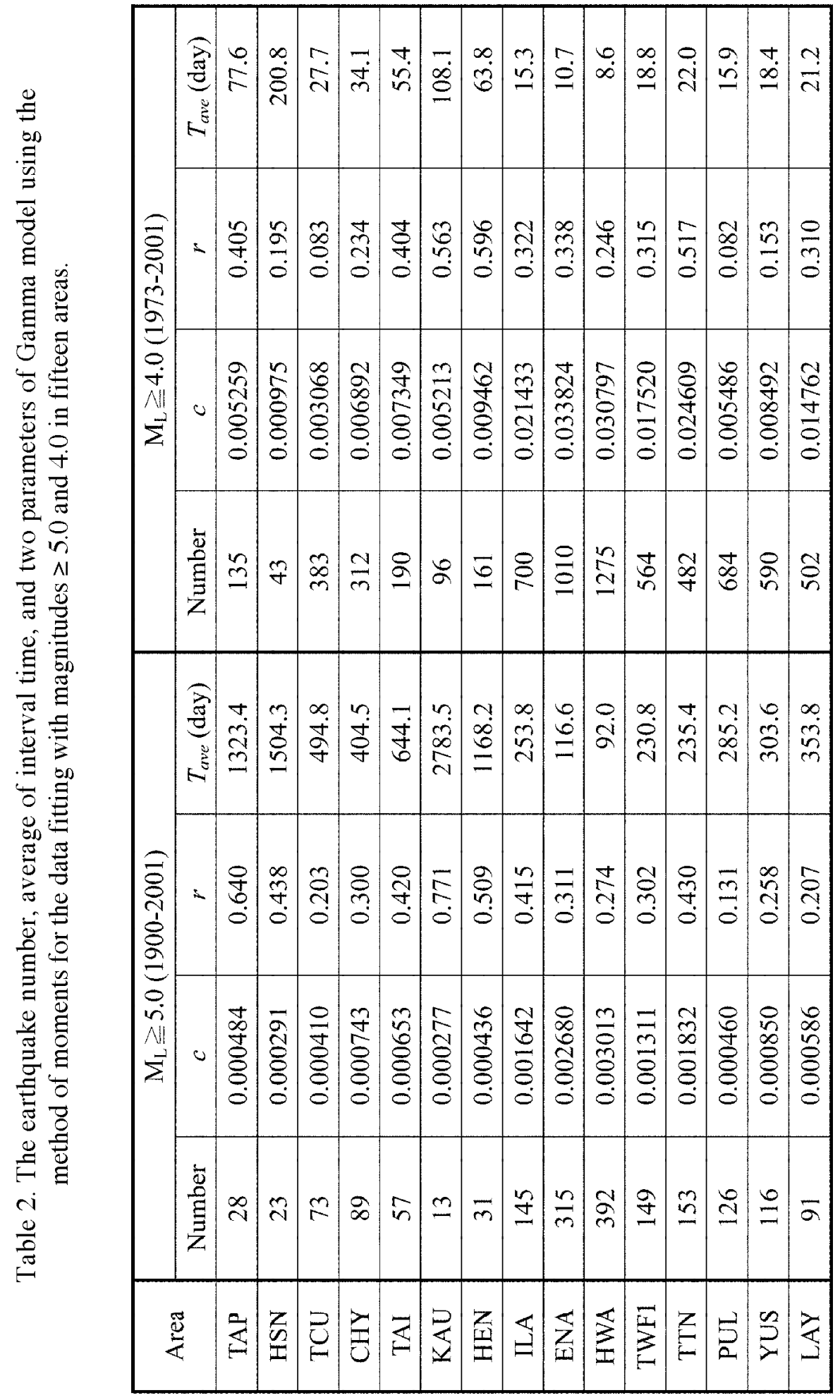


$\geq 4.0$ and 5.0 using the method of moments for data fitting in the fifteen areas. The results are given in Table 2. The average interval time $\left(T_{\text {ave }}\right)$ for earthquakes with magnitude $\geq 5.0$ is large in KAU, HSN, TAP, and HEN areas. The corresponding numbers of event are small. In contrast, small $T_{\text {ave }}$ values appear in HWA, ENA, TWF1, TTN, and ILA areas that are in eastern Taiwan. The corresponding numbers of event are large. The relationship between the average interval time and number of events is inversely proportional. Similar results are also obtained for earthquakes with magnitude $\geq 4$.0. Figures $9 \mathrm{a}, \mathrm{c}$ show in a logarithmic-logarithmic plot the inverse relationship between $T_{\text {ave }}$ and $c$ for earthquakes with magnitudes $\geq 5.0$ and 4 . 0 , respectively. Regression of $T_{\text {ave }}$ vs $c$ yields the following equations:

$$
\begin{aligned}
& \ln \left(T_{\text {ave }}\right)=-1.0964 \ln (c)-1.7692 \text { for } \mathrm{M} \geq 5.0, \\
& \ln \left(T_{\text {ave }}\right)=-0.7376 \ln (c)-0.0388 \text { for } \mathrm{M} \geq 4.0 .
\end{aligned}
$$

The relationships between $T_{\text {ave }}$ and $r$ for magnitudes $\geq 5.0$ and 4.0 are shown in Figs. 9b, d, respectively. The $r$ values for PUL, TCU, and YUS areas are significantly lower than in other areas. The $r$ value in all areas is less than 1, showing clustering of earthquakes in the whole of Taiwan.

The contour map of parameter $c$ for earthquakes with magnitude $\geq 5.0$ using the method of moments for data fitting in the fifteen areas is shown in Fig. 10a. The $c$ values fall in the range $2.77 \times 10^{-4}$ to $3.013 \times 10^{-3}$. Large $c$ values appear in eastern coastal areas indicating earthquakes occur more frequently in these areas. This result is consistent with the seismicity distribution of Taiwan (Fig. 3). The lower $c$ values appear in northwestern Taiwan, where earthquakes occurred sparsely. Figure 11a shows a contour map of the parameter $r$ of the Gamma model. The $r$ values fall in the range 0.131 to 0.771 . The unusually low $r$ value in central Taiwan was caused by the Chi-Chi earthquake, because many aftershocks occurred in central Taiwan after the Chi-Chi earthquake. It can be explained by Fig. 12 which shows the results with magnitudes $\geq 4.0$ and 5.0 in the TCU area for the periods before the Chi-Chi earthquake and for all times. Similar results are also obtained in YUS and PUL areas. We also repeat the calculation for magnitude $\geq 4.0$ for the period from 1973 to 2001. The two parameters of the Gamma model show a short-term pattern for moderate earthquakes. The contour map of parameter with magnitude $\geq 4.0$ is shown in Fig. 10b. The ranges of $c$ value are from $9.75 \times 10^{-4}$ to $3.3824 \times 10^{-2}$. In spite of the difference in $c$ values by an order of magnitude between Figs. 10a and b, the patterns of the contour maps are similar. Clearly, earthquakes occur in eastern Taiwan much more frequently than in western Taiwan. The contour map of parameter $r$ with magnitude $\geq 4.0$ is shown in Fig. 11b. The $r$ values fall in the range 0.082 to 0.596 . The $r$ values for magnitude $\geq 4.0$ are smaller than the $r$ values for magnitude $\geq 5.0$. The clustering phenomenon is evident for smaller earthquakes with magnitude $\geq 4.0$.

We cannot use the earthquake data with magnitude $\geq 6.0$ to determine the $c$ and $r$ values for individual areas because the database is incomplete for such an analysis. Instead, we can analyze the earthquake data with magnitude $\geq 6.0$ for the whole of Taiwan. Figure 13 shows the observed data along with the Gamma model fitting using the method of moments for 
(a)

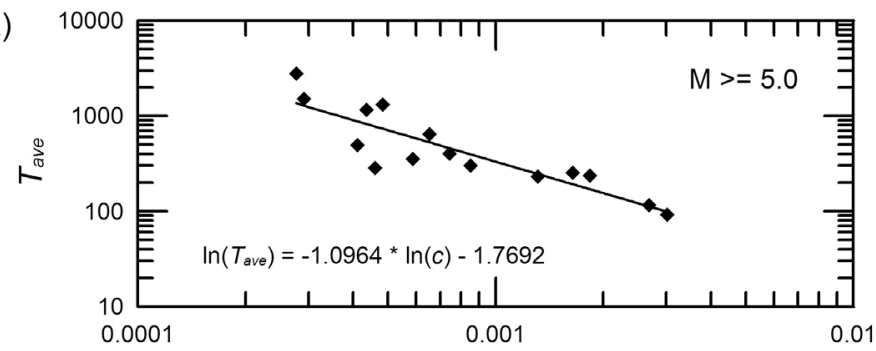

(b)

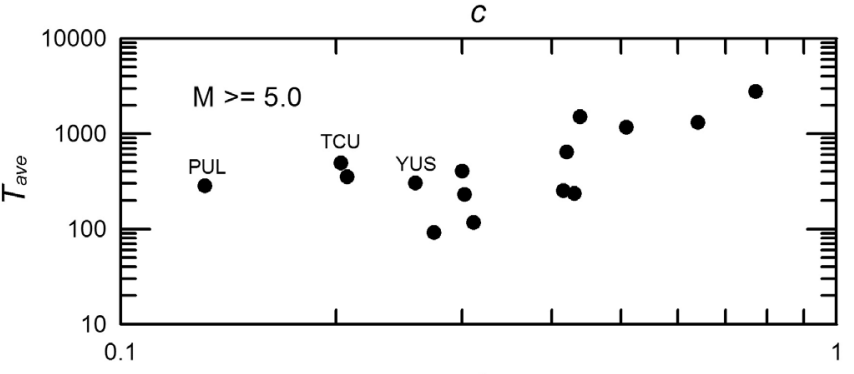

(c)

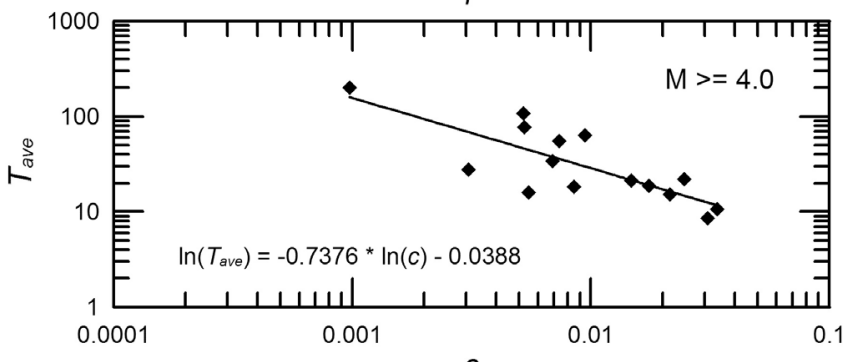

(d)

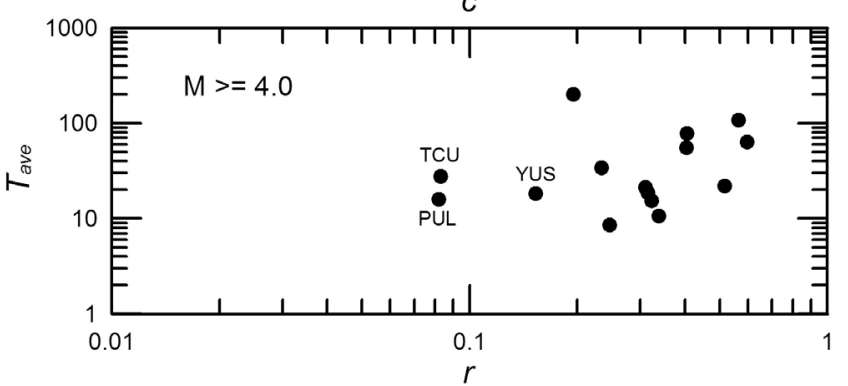

Fig. 9. (a) The relationship between average interval time $\left(T_{\text {ave }}\right)$ and parameter $c$ from the Gamma model using the method of moments for earthquake magnitude $\geq 5.0$. (b) The relationship between average interval time and parameter $r$ from the Gamma model using the method of moments for earthquake magnitude $\geq 5.0$. (c) The relationship between average interval time and parameter $c$ from the Gamma model using the method of moments for earthquake magnitude $\geq 4.0$. (d) The relationship between average interval time and parameter $r$ from the Gamma model using the method of moments for earthquake magnitude $\geq 4.0$. 
(a)

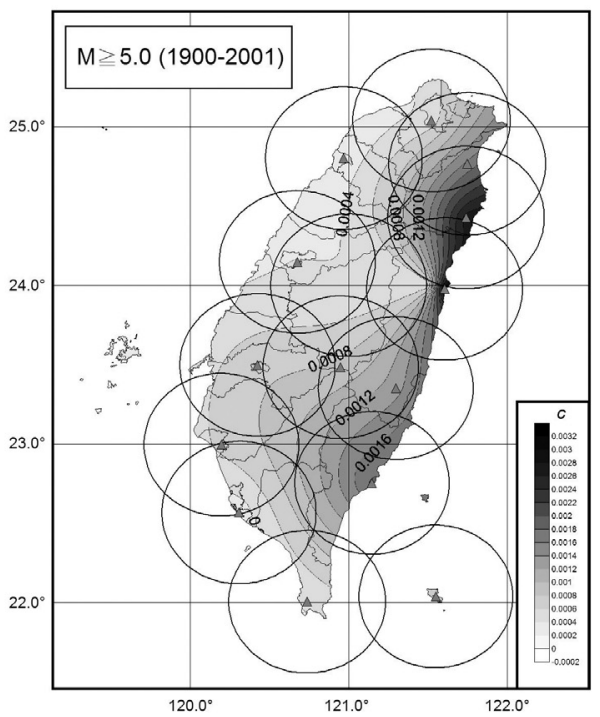

(b) c values

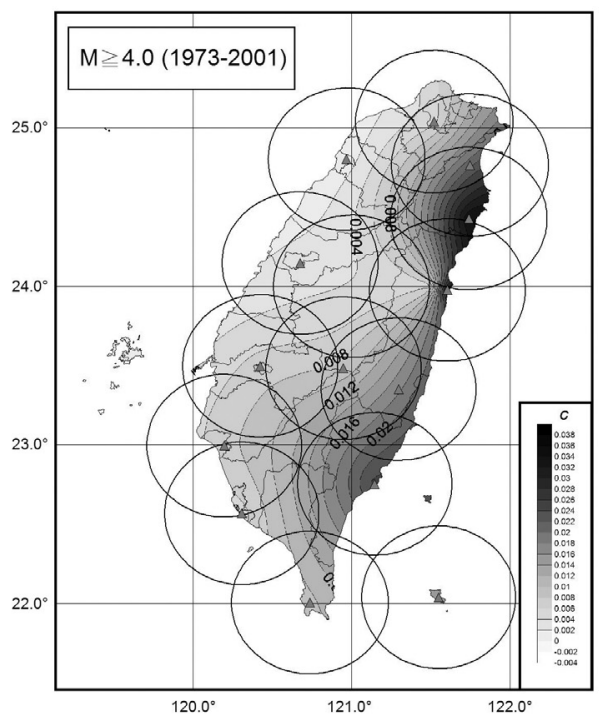

Fig. 10. The contour map of $c$ value of the Gamma model for magnitude $\geq 5.0$ (a) and for magnitude $\geq 4.0$ (b) in Taiwan.

(a)

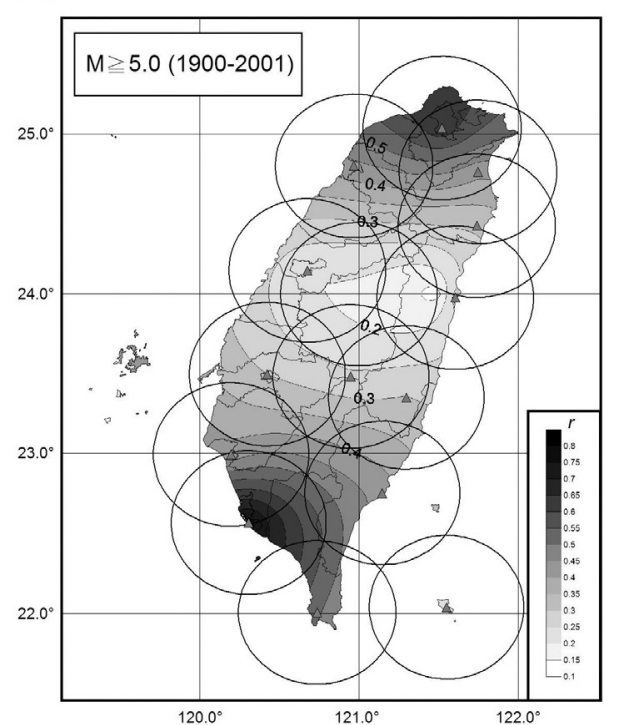

(b) rvalues

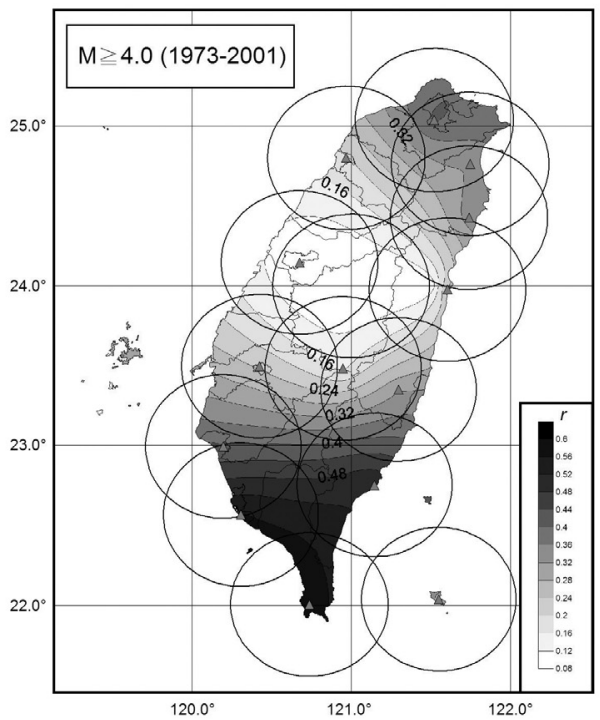

Fig. 11. The contour map of $r$ value of the Gamma model for magnitude $\geq 5.0$ (a) and for magnitude $\geq 4.0$ (b) in Taiwan. 
Fig. 12. The relationship of probability and interval time for magnitudes $\geq 4.0$ and $\geq 5.0$ in the TCU area for the periods before the Chi-Chi earthquake and of all periods. The lines show the corresponding fittings from the Gamma model using method of moments.

earthquakes with magnitude $\geq 6.0$ in the whole of Taiwan. From this figure we can see at 50 percent probability of occurrence the interval time is 75 days. The probability increases as the interval time increases. The probability goes up to 70 percent at a recurrence time of about 200 days. We have shown above a method to construct the recurrence model based on seismicity data. This model can be used to forecast the probability of earthquake recurrence in the future. 


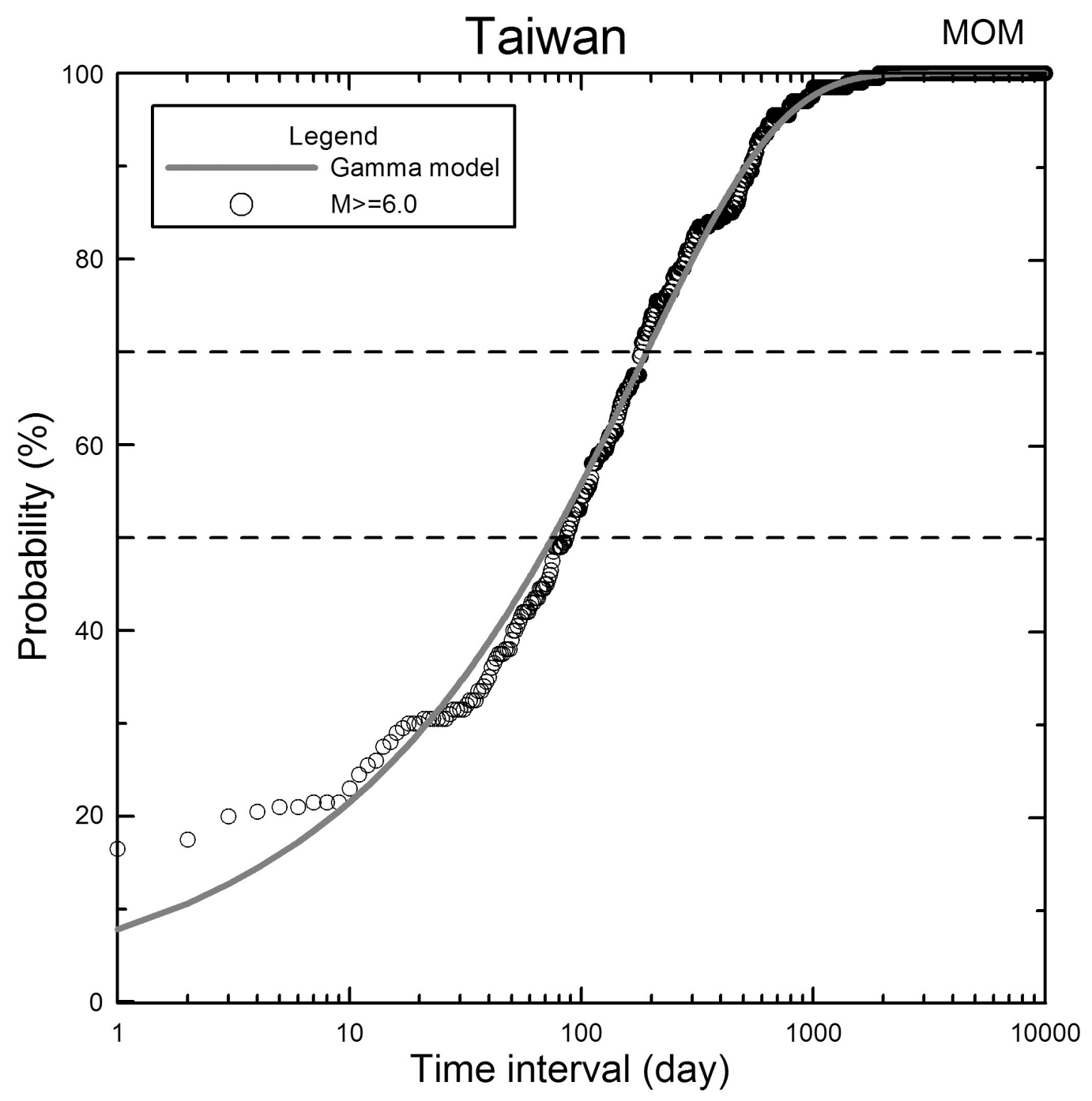

Fig. 13. The relationship of probability and interval time for earthquake magnitude $\geq 6.0$ in the whole of Taiwan. The corresponding fitting is from the Gamma model using the method of moments.

\section{DISCUSSIONS AND CONCLUSIONS}

Previous studies have shown different recurrence models are best suited for different sets of data (Utsu 1984; Parvez and Ram 1997; Shimazaki 2002). We can specify the areas that are best suited for a certain recurrence model based on the data sets. Many researchers consider earthquake occurrence as a Poisson process (Merz and Cornell 1973). We have examined the distribution of earthquake recurrence intervals in Taiwan using four recurrence models. The 
results show that the Gamma model using the method of moments gives the best fit to the data over other recurrence models. It means the Gamma model is suitable for analyzing the earthquake catalog of Taiwan. In this study, we have not declustered the earthquake catalog. We have taken the whole seismicity data set for analysis of the Gamma recurrence model.

We can use the two parameters of the Gamma model to represent the characteristics of seismicity patterns. For example, the parameter $c$ values are significantly higher in eastern Taiwan than in western Taiwan indicating earthquakes occur more frequently in eastern Taiwan. The parameter $r$ values are lowest in central Taiwan. This was due to the clustering of numerous aftershocks in central Taiwan following the Chi-Chi earthquake. We have divided Taiwan into fifteen circular areas. The results show distinct the characteristics of each area based solely on past seismicity. The recurrence probability depends on the model parameters ( $c$ and $r$ ) and the time since the last event. So we can use the recurrence model as a means to forecast future earthquakes in an area.

Acknowledgements We would like to thank the Central Weather Bureau of Taiwan for providing the earthquake catalog data. We also thank Prof. Ta-Liang Teng and another anonymous reviewer for their constructive suggestions and comments. We acknowledge Dr. ChienHsin Chang for his information about the CWB earthquake catalog. This work was supported by National Science Council under Grant NSC 91-2116-M-008-010, and by the Ministry of Education under Grant 91-N-FA07-7-4.

\section{REFERENCES}

Cheng, S. N., and Y. T. Yeh, 1989: Catalog of the earthquakes in Taiwan from 1604 to 1988. Inst. Earth Sci., Acad. Sinica Open-File Rept. IES-R-661, 255 p. (in Chinese)

Goes, S. D. B., 1996: Irregular recurrence of large earthquakes: an analysis of historic and paleoseismic catalogs. J. Geophys. Res., 101, 5739-5749.

Gómez, J. B., and A. F. Pacheco, 2004: The minimalist model of characteristic earthquakes as a useful tool for description of the recurrence of large earthquakes. Bull. Seism. Soc. Am., 94, 1960-1967.

Gutenberg, B., and C. F. Richter, 1944: Frequency of earthquakes in California. Bull. Seism. Soc. Am., 34, 185-188.

Gutenberg, B., and C. F. Richter, 1949: Seismicity of the earth and associated phenomena, Princeton Univ. Press.

Hagiwara, Y., 1974: Probability of earthquake occurrence as obtained from a Weibull distribution analysis of crustal strain. Tectonophys., 23, 313-318.

Matthews, M. V., W. L. Ellsworth, and P. A. Reasenberg, 2002: A Brownian model for recurrent earthquakes. Bull. Seism. Soc. Am., 92, 2233-2250.

Merz, H. A., and C. A. Cornell, 1973: Seismic risk analysis based on a quadratic magnitudefrequency law. Bull. Seism. Soc. Am., 63, 1999-2006.

Nishenko, S. P., and R. Buland, 1987: A generic recurrence interval distribution for earthquake forecasting. Bull. Seism. Soc. Am., 77, 1382-1399. 
Parvez, I. A., and A. Ram, 1997: Probabilistic assessment of earthquake hazards in the northeast Indian peninsula and Hindukush regions. Pure Applied Geophys., 149, 731-746.

Rikitake, T., 1991: Assessment of earthquake hazard in the Tokyo area, Japan.Tectonophys., 199, 121-131.

Shimazaki, K., 2002: Long-term probabilistic forecast in Japan and time-predictable behavior of earthquake recurrence. In: Fujinawa Y., and A. Yoshida, (Eds.), Seismotectonics in Convergent Plate Boundary, TERRAPUB, 37-43.

Shin, T. C., 1993: The calculation of local magnitude from the simulated Wood-Anderson seismograms of the short-period seismograms in Taiwan area. Terr. Atmos. Ocean. Sci., 4, 155-170.

Sykes, L. R., and S. P. Nishenko, 1984: Probabilities of occurrence of large plate rupturing earthquakes for the San Andreas, San Jacinto, and Imperial faults, California, 19832003. J. Geophys. Res., 89, 5905-5927.

Udías, A., and J. Rice, 1975: Statistical analysis of microearthquake activity near San Andreas geophysical observatory, Hollister, California. Bull. Seism. Soc. Am., 65, 809-827.

Utsu, T., 1984: Estimation of parameters for recurrence models of earthquakes. Bull. Earthquake Res. Inst. Univ. Tokyo, 59, 53-66.

Utsu, T., 2002: Statistical features of seismicity. In: Lee, W. H. K., H. Kanamori, P. C. Jennings, and C. Kisslinger, (Eds.), International Handbook of Earthquake \& Engineering Seismology, 81A, Academic Press, 719-732.

Wang, J. H., 1992: Magnitude scale and their relations for Taiwan earthquakes: a review. Terr. Atmos. Ocean. Sci., 3, 449-468.

Yeh, Y. T., S. N. Cheng, T. C. Shin, and M. Y. Ho, 1995: Assessment of earthquake location and magnitude for several Taiwan catalogs (III). Central Wea. Bureau Tech. Report., 11, 243-264. (in Chinese) 